\title{
Citalopram for hot flashes
}

Citalopram, a selective serotonin reuptake inhibitor, is an effective, well-tolerated agent for the management of hot flashes, a novel study published in the Journal of Clinical Oncology has determined.

In menopause, up to $75 \%$ of women experience hot flashes, which can negatively impact quality of life. Many cancer survivors cannot or do not wish to receive estrogen-based treatment for menopausal symptoms, increasing the need for good alternatives that are effective without unwanted adverse effects. "As the definitive physiology of hot flashes is not known, I believe it cannot be assumed that the efficacy of the new antidepressants for hot flashes is a class effect," says lead researcher Debra L. Barton from the Mayo Clinic (Rochester, MN, USA). "Instead, individual agents need to be evaluated."

Previous studies with citalopram were promising. In one trial, women treated unsuccessfully with the serotonin-norepinephrine reuptake inhibitor venlafaxine, were crossed over to citalopram therapy, and hot flashes were reduced by $50 \%$. Nevertheless, other trials found no sign of efficacy of citalopram on the reduction of hot flashes. In a randomized, double-blind phase III trial, Barton and colleagues evaluated citalopram versus placebo for 6 weeks in 254 postmenopausal women with a minimum of 14 hot flashes per week. The study design made use of a prospective hot flash diary.Participants recorded their hot flashes in real time, meaning, they marked down as and when they experienced each hot flash. At the end of every $24 \mathrm{~h}$ period, the women added up the noted flashes, which provided an accurate account of their total numbers. All women recorded hot flashes for 7 days before starting treatment and were then titrated to citalopram at daily target doses of $10 \mathrm{mg}, 20 \mathrm{mg}$ or $30 \mathrm{mg}$ versus placebo to evaluate the change in hot flash score from baseline to 6 weeks.

No difference in hot flash improvement was found between the different doses of citalopram, but citalopram was significantly better than placebo. Reductions in mean hot flash scores were 2.0 (23\%) for placebo compared with 7.0 (49\%), 7.7 (50\%) and 10.7 (55\%) for $10 \mathrm{mg}, 20 \mathrm{mg}$ and $30 \mathrm{mg}$ of citalopram, respectively. Hence, as little as $10 \mathrm{mg}$ of citalopram was efficient in reducing hot flashes $>45 \%$. Furthermore, the treatment was well-tolerated, with no marked adverse effects reported. The most marked improvement with regards to the Hot Flash Related Daily Interference scale, which is used to assess the effect of hot flashes on quality of life, was determined in the group of women treated with $20 \mathrm{mg}$ citalopram. This increased dose of citalopram provided substantial benefits in many areas of life, including work, sleep and mood. However, a dose of $30 \mathrm{mg}$ citalopram daily "is clearly not needed as it did not result in greater reductions of hot flashes, yet increased the number of adverse effects," states Barton. She plans to continue her work by "combining low-dose pharmacologic with nonpharmacologic interventions, to improve efficacy and keep unwanted adverse effects to a minimum".

Taken together, although no marked dose response can be determined at a dose $>10 \mathrm{mg}$ daily, citalopram has additional beneficial effects on quality of life when administered at a dose of $20 \mathrm{mg}$ per day. "Citalopram is a low-cost, low-toxicity, effective agent for reducing bothersome hot flashes," concludes Barton.

Linda Koch

Original article Barton, D. L. et al. Phase III, placebo-controlled trial of three doses of citalopram for the treatment of hot flashes: NCCTG trial N05C9. J. Clin. Oncol. 28, 3278-3283 (2010) 